\title{
Obesidade infantil: Uma problemática da sociedade atual
}

\author{
Childhood obesity: A problem in today's society \\ Obesidad infantil: Um problema em la sociedade actual
}

Recebido: 24/05/2021 | Revisado: 29/05/2021 | Aceito: 29/05/2021 | Publicado: 02/06/2021

Pedro Victor Ramos Zigarti

ORCID: https://orcid.org/0000-0002-1188-9816

Centro Universitário Fametro, Brasil

E-mail: pedrozigarti@gmail.com

Idelmar da Silva Barata Junior

ORCID: https://orcid.org/0000-0001-6512-4854

Centro Universitário Fametro, Brasil

E-mail: idelmarbarata@hotmail.com

José Carlos de Sales Ferreira

ORCID: https://orcid.org/0000-0002-1867-8229

Centro Universitário Fametro, Brasil

E-mail: jcarlos.sales@gmail.com

\begin{abstract}
Resumo
Introdução: É observado ao decorrer das últimas décadas que houve um crescimento no mundo todo da obesidade infantil, e devido ao seu evidente crescimento ela vem se caracterizando como uma nova epidemia mundial Objetivo: analisar os aspectos que cercam a obesidade infantil e a sua prevalência na sociedade atual. Metodologia: Este estudo trata-se de uma pesquisa de revisão integrativa da literatura. Resultados e Discussão: A obesidade, já na infância, está relacionada a várias complicações, como também a uma maior taxa de mortalidade. E, quanto mais tempo o indivíduo se mantém obeso, maior é a chance de as complicações ocorrerem, assim como mais precocemente. Conclusão: Ações no âmbito coletivo devem envolver políticas públicas que promovam a saúde, o bem-estar e a qualidade de vida das populações.
\end{abstract}

Palavras-chave: Obesidade; Infantil; Sociedade.

\begin{abstract}
Introduction: It is observed over the last few decades that there has been a growth in the world of childhood obesity, and due to its evident growth it has been characterized as a new worldwide epidemic Objective: to analyze the aspects surrounding childhood obesity and its prevalence in current Society. Methodology: This study is an integrative literature review research. Results and Discussion: Obesity, already in childhood, is related to several complications, as well as to a higher mortality rate. And, the longer the individual remains obese, the greater the chance of complications occurring, as well as earlier. Conclusion: Actions at the collective level must involve public policies that promote the health, well-being and quality of life of the populations.
\end{abstract}

Keywords: Obesity; Children; Society.

\section{Resumen}

Introducción: Se observa en las últimas décadas que ha habido un crecimiento en el mundo de la obesidad infantil, y por su evidente crecimiento se ha caracterizado como una nueva epidemia mundial Objetivo: analizar los aspectos que rodean la obesidad infantil y su prevalencia en la sociedad actual. Metodología: Este estudio es una investigación de revisión de literatura integradora. Resultados y Discusión: La obesidad, ya en la infancia, está relacionada con varias complicaciones, así como con una mayor tasa de mortalidad. Y, cuanto más tiempo el individuo permanezca obeso, mayor será la posibilidad de que ocurran complicaciones, así como antes. Conclusión: Las acciones a nivel colectivo deben involucrar políticas públicas que promuevan la salud, el bienestar y la calidad de vida de las poblaciones.

Palabras clave: Obesidad; Niño; Sociedad.

\section{Introdução}

É observado ao decorrer das últimas décadas que houve um crescimento no mundo todo da obesidade infantil, e devido ao seu evidente crescimento ela vem se caracterizando como uma nova epidemia mundial, que atinge tanto países desenvolvidos, como aqueles que estão em desenvolvimento, afetando o desenvolvimento e fatores como a qualidade de vida das crianças como um todo (Diez et al., 2016). 
Nesse seguimento, é referido por Monteiro et al. (2015), que a obesidade não é recente na história da humanidade, porém nunca tinha alcançado dados tão alarmantes como na atualidade, tornando-se um grande problema de saúde pública. A estimativa mundial da International Obesity Task Force (IOTF, 2005) é de que haja, atualmente, 155 milhões de crianças com excesso de peso, seja este sobrepeso ou obesidade, tendo como os países de maior prevalência os industrializados.

É afirmado por Anjos et al. (2016), que a obesidade infantil também tem crescido evidentemente nas últimas três décadas e que vem se tornando um efetivo problema de saúde pública. As causas de isso acontecer estão ligadas às mudanças no estilo de vida e nos hábitos alimentares, pois cada vez mais se faz consumo de alimentos industrializados, geralmente com alto teor calórico à custa de gordura saturada e colesterol.

Sendo assim, nota-se que é consenso que a obesidade infantil vem aumentando de forma significativa e que ela determina várias complicações na infância e na idade adulta. Na infância, o manejo pode ser ainda mais difícil do que na fase adulta, pois está relacionado a mudanças de hábitos e disponibilidade dos pais, além de uma falta de entendimento da criança quanto aos danos da obesidade (Leão et al., 2017).

A alta prevalência de Excesso de Peso Corporal que muitas vezes já é proveniente da fase infantil pode levar na vida adulta a um aumento das morbidades associadas a este distúrbio como a diabetes, hipertensão arterial, dislipidemia, hipercolesterolêmia, doenças cardiovasculares e ao desenvolvimento da síndrome metabólica, onde diminui a qualidade de vida e afeta a saúde da pessoa diretamente (Bras et al., 2017).

Por esse motivo, é essencial a instituição de medidas para a prevenção da obesidade ainda na infância. Essas medidas podem ser iniciadas já na vida intrauterina, a partir da promoção da saúde da gestante, identificação de fatores de risco para sua saúde e da criança e da orientação quanto a hábitos de vida que favoreçam o peso saudável (Baladan et al., 2016).

É afirmado por Abrantes et al. (2018), que durante os primeiros anos de vida, é importante a orientação alimentar adequada e específica para que os cuidadores entendam não apenas quais são os alimentos que devem ser consumidos, mas também sua forma de preparo, quantidade e idade em que devem ser introduzidos. Além disso, é importante que a família, a escola e a comunidade como um todo estejam engajados no projeto coletivo de melhorar a saúde da população infantil.

É perceptível que atualmente a obesidade é tida como uma das doenças crônicas não transmissíveis mais comuns na infância, com tendência de se prolongar até a vida adulta, tornando precoce o surgimento de outras doenças a ela associadas, tais como hipertensão arterial, dislipidemia, diabetes tipo 2, entre outros fatores de risco que podem se apresentar (Santos $e t$ al., 2020; Barreto \& Nunes, 2019).

Com isso, o desenvolvimento desse estudo justifica-se pela necessidade da sociedade atual em conhecer acerca da obesidade infantil e de quanto esta é danosa para a saúde da criança, sendo ainda de extrema importância os hábitos alimentares que são estimulados a estas crianças.

\section{Metodologia}

Este estudo trata-se de uma pesquisa de revisão integrativa da literatura. Tendo estes tipos de pesquisas descritivas como objetivo primordial a descrição das características de determinada população ou fenômeno, e as pesquisas de caráter exploratório tem como objetivo proporcionar uma maior familiaridade com o problema, com vista a torná-lo mais explícito (Silva et al., 2010).

A Revisão Integrativa é um método de pesquisa apontado como ferramenta de grande relevância no campo da saúde, por proporcionar a busca, a avaliação crítica e a síntese de evidências sobre um tema investigado. Esses aspectos facilitam a identificação dos resultados relevantes, de lacunas que direcionam para o desenvolvimento de futuras pesquisas e auxiliam o profissional a escolher condutas e a tomar decisões, proporcionando um saber crítico (Silva et al., 2010). 
A coleta das informações para a pesquisa bibliográfica será por meio da exploração da base de dados da Biblioteca Virtual em Saúde (BVS), Biblioteca Cientifica Eletrônica Virtual (SCIELO) e Literatura Latino - Americana e do Caribe em Ciências da Saúde (LILACS). A busca na base de dados foi orientada pelas palavras-chave: obesidade infantil; crianças; sobrepeso; e será realizado em todos os índices, buscando captar o maior número de artigos publicados no período proposto que abordem a temática em discussão.

Para uma análise crítica e reflexiva dos estudos incluídos na revisão, será realizada uma leitura minuciosa e criteriosa destacando os que atingirem os critérios de inclusão e que contemplarem o objetivo proposto, para viabilizar o resultado da pesquisa de forma clara e objetiva. Serão incluídos os artigos publicados em língua portuguesa nos últimos dez anos, com texto completo, disponível on-line, com acesso livre. Serão excluídos da amostra os artigos publicados em línguas estrangeiras, os que não apresentarem o texto na integra, artigos que não apresentavam relação direta com o tema, resumos, monografias, dissertações, teses e artigos repetidos

\section{Resultados e Discussão}

\subsection{Obesidade Infantil: epidemiologia}

Recentemente, importante estudo realizado no Brasil pelo Instituto Brasileiro de Geografia e Estatística (IBGE) em parceria com o Ministério da Saúde, a mais recente Pesquisa de Orçamentos Familiares (POF 2008-2009), analisou características nutricionais e antropométricas e demonstrou alterações no perfil da população brasileira nas últimas quatro décadas (Monteiro, 2015).

Os resultados evidenciaram que o déficit de altura (importante indicador de desnutrição) caiu de 29,3\% (1974-75) para 7,2\% (2008-09) entre os meninos e de 26,7\% para 6,3\% entre as meninas, na idade de cinco a nove anos. Em contraste, o excesso de peso (incluindo a obesidade) apresentou altos índices de prevalência em 2008-09: 51,4\% entre os meninos e 43,8\% entre as meninas, na mesma faixa etária de cinco a nove anos, demonstrando a relevância desse problema para a saúde pública brasileira (Grilo, 2016).

$\mathrm{O}$ excesso de peso e a obesidade apresentam alta taxa de prevalência, que vem aumentando ao longo do tempo. A análise desses dados revela que em 2008-09, entre as crianças de cinco a nove anos de idade, 32\% das meninas se encontravam com sobrepeso e 11,8\% eram obesas. Já para o sexo masculino, as taxas eram ainda superiores, de 34,8 e 16,6\%, respectivamente (Wang, 2018).

Entre os meninos, $21,7 \%$ apresentavam sobrepeso e 5,9\% obesidade. Já no sexo feminino, os índices de sobrepeso e obesidade foram de 19,4 e 4\%, respectivamente. Ainda que mais baixos em relação àqueles referentes à idade de cinco a nove anos, esses índices são ainda altos, especialmente quando comparados aos de 1974-75, quando eram bem mais reduzidos (Toddei, 2018).

No Brasil, a prevalência desse tipo de obesidade entre crianças e adolescentes tem aumentado rapidamente nos últimos anos, devido, principalmente, ao estilo de vida inadequado adquirido pela maior parte da população. Nos últimos 30 anos, houve mudança perceptível no padrão alimentar familiar, com o aumento do consumo de fast-food e alimentos ricos em gordura, sal e açúcar (Hammer, 2015).

\subsection{Fatores de Risco para Obesidade Infantil}

A quantidade total de gordura, o excesso de gordura em tronco ou região abdominal e o excesso de gordura visceral são três aspectos da composição corporal associados à 5 ocorrência de doenças crônico-degenerativas. $\mathrm{O}$ aumento do colesterol sérico é um fator de risco para doença coronariana, e esse risco é ainda maior quando associado à obesidade (Must, 2017). 
A obesidade é fator de risco para dislipidemia, promovendo aumento de colesterol, triglicerídeos e redução da fração HDL colesterol. A perda de peso melhora o perfil lipídico e diminui o risco de doenças cardiovasculares. A qualidade da ingestão é um fator de risco para doença coronariana e que a ingestão da criança está intimamente relacionada com a dos pais (Cole, 2016).

A obesidade, já na infância, está relacionada a várias complicações, como também a uma maior taxa de mortalidade. E, quanto mais tempo o indivíduo se mantém obeso, maior é a chance de as complicações ocorrerem, assim como mais precocemente. A obesidade, já na infância, está relacionada a várias complicações, como também a uma maior taxa de mortalidade. E, quanto mais tempo o indivíduo se mantém obeso, maior é a chance de as complicações ocorrerem, assim como mais precocemente (Klish, 2018).

Vários fatores influenciam o comportamento alimentar, entre eles fatores externos (unidade familiar e suas características, atitudes de pais e amigos, valores sociais e culturais, mídia, alimentos rápidos, conhecimentos de nutrição e manias alimentares) e fatores internos (necessidades e características psicológicas, imagem corporal, valores e experiências pessoais, autoestima, preferências alimentares, saúde e desenvolvimento psicológico) (Toriano, 2018).

Dificuldade em estabelecer um bom controle de saciedade é um fator de risco para desenvolver obesidade, tanto na infância quanto na vida adulta. Quando as crianças são obrigadas a comer tudo o que é servido, elas podem perder o ponto da saciedade. A saciedade se origina após o consumo de alimentos, suprime a fome e mantém essa inibição por um período de tempo determinado (Tanner, 2016).

O público infantil é o mais vulnerável aos apelos promocionais. Entre as diversas formas de influência sobre as práticas alimentares provenientes do meio, a mídia, nas suas múltiplas formas, está entre aquelas que mais rapidamente estão assumindo papel central na socialização de crianças e jovens (Monteiro, 2015).

Publicidade e propaganda são técnicas largamente usadas pelas empresas para encorajar o consumo de seus produtos. As indústrias investem pesadamente divulgando fast-food ricos em calorias, bebidas carbonatadas, cereais açucarados matinais e snacks, alimentos os quais tendem a ser ricos em gorduras, açúcar e sal, bem como pobre em nutrientes (Tanner, 2016).

As propagandas de alimentos na televisão são aquelas que têm recebido maior atenção dos pesquisadores na atualidade. Várias pesquisas no exterior e no Brasil comprovam a relação entre os comerciais de produtos alimentícios, cuja exposição é feita nos horários de maior audiência da televisão, e o comportamento alimentar dos consumidores, principalmente das crianças (Cole, 2016).

\subsection{Medidas preventivas para a Obesidade Infantil}

Orientações Nutricionais Como os programas de intervenção ainda têm pouco consenso, a prevenção continua sendo o melhor caminho. Os esforços para a prevenção da obesidade na infância são provavelmente mais eficazes quando endereçados simultaneamente aos alvos primordial, primário e secundário, com metas apropriadamente diferentes (Gray, 2019).

A prevenção primordial visa prevenir que as crianças se tornem de risco para sobrepeso; a prevenção primária objetiva evitar que as crianças de risco adquiram sobrepeso; 6 e a prevenção secundária visa impedir a gravidade crescente da obesidade e reduzir a comorbidade entre crianças com sobrepeso e obesidade. Dentro deste cenário, as prioridades básicas de ação podem ser identificadas, priorizadas e vinculadas às estratégias de intervenção potencialmente satisfatórias (Diaz, 2016).

As iniciativas de prevenção primordial e primária são as mais eficazes, provavelmente se forem iniciadas antes da idade escolar e mantidas durante a infância e a adolescência. Deve haver um esforço significativo no sentido de direcioná-las à 
prevenção da obesidade já na primeira década de vida. A política da escola pode promover ou desencorajar dietas saudáveis e atividade física (Daniels, 2017).

Em nosso meio, a obesidade infantil é um sério problema de saúde pública, que vem aumentando em todas as camadas sociais da população brasileira. É um sério agravo para a saúde atual e futura dos indivíduos. Prevenir a obesidade infantil significa diminuir, de uma forma racional e menos onerosa, a incidência de doenças crônico-degenerativas (Oliveira, 2015).

Para alcançar uma alimentação saudável, além de fornecer informações corretas sobre alimentação e saúde (promoção), é preciso evitar que informações incorretas e contraditórias alcancem indivíduos (proteção) e, ao mesmo tempo, propiciar a esses indivíduos condições que tornem factíveis a adoção das orientações que recebem (apoio) (Fontanive, 2017).

\section{Conclusão}

A obesidade pode ser controlada para esse ter o acesso ao método e o profissional correto para uma avaliação e mudar os hábitos alimentares associados à atividade física, eliminando assim as predisposições a futuras enfermidades. Destacando-se que esta é decorrente de uma série de fatores, porém, os principais é o consumo exagerado de alimentos não saudáveis, tais como: alimentos açucarados e ultra processados, associados ao sedentarismo e também de multifatorial, que são fatores ambientais e genéticos que podem ser associados no diagnóstico.

Ações no âmbito coletivo devem envolver políticas públicas que promovam a saúde, o bem-estar e a qualidade de vida das populações onde a parceria entre o governo e a sociedade civil seria um caminho bastante promissor na prevenção e tratamento da obesidade, por meio da responsabilização e do autocuidado, permitindo que a comunidade participe do processo de promoção da saúde.

\section{Referências}

Ascari, R. A., Souza, L. A., Ferraz, L., Silva, O. M. \& Adamy, E. K. (2012). Obesidade Infantil: um olhar dos enfermeiros inseridos na Atenção Básica. Associação Brasileira de Enfermagem.

Araújo, S. N. M., et al. (2012). Obesidade infantil: Conhecimento e práticas de enfermeiros da Atenção Básica. Enfermagem em foco. 3(3), 139-42.

Auwerx, J. \& Staels B. Leptin. Lancet. (1998), 351(9104), 737-42.

Azevedo, C.M, et al. (2016). Food environments in schools and in the immediate vicinity are associated with unhealthy food consumption among Brazilian adolescents. Prev Med, 88, 73-79.

Barreto, D. V. \& Nunes, C. P. (2019). Efeitos da dieta e exercício físico na prevenção da obesidade infantil. Revista de Medicina de Família e Saúde Mental, $1(2)$.

Barlow SE, Dietz WH, Klish WJ. \& Trowbridge FL. (2018). Medical Evaluation of overweight children and adolescents: reports from pediatricians, pediatric nurse practitioners, and registered dietitians. Pediatrics. 110(1), 222-8.

Barlow SE, Trowbridge FL, Klish WJ. \& Dietz WH. (2002). Treatment of child and adolescent obesity: reports from pediatricians, pediatric nurse practitioners, and registered dietitians. Pediatrics. 110, 229- 235.

Brasil, Ministério Da Saúde. (2012). Secretaria de Atenção à Saúde. Departamento de Atenção Básica. Política Nacional de Alimentação e Nutrição.

Batista, Mondini \& Jaime. (2014). Ações do programa saúde na escola e da alimentação escolar na prevenção do excesso de peso infantil. Epidemiologia e serviços de saúde.

Beltramin, S. M., et al. (2015). Influência da mídia na obesidade infantil. Revista Digital. Buenos Aires.

Botelho, E. G., Soares, W. D. \& Finelli, L. (2019). Hábitos alimentares em crianças e sua associação comobesidade e sobrepeso. Recital Revista de Educação, Ciência e Tecnologia de Almenara/MG, 1(1), 24-36.

Burlandy, L., et al. (2016). Políticas de promoção da saúde e potenciais conflitos de interesses que envolvem o setor privado comercial. Cien Saude Colet. 21(6), 1809-1818.

Casemiro, Fonseca \& Secco. (2014). Promover saúde na escola: reflexões a partir de uma revisão sobre saúde escolar na América Latina. Rev. brasileira de enfermagem, 10 .

Ceccatto, D., et al. (2018). A influência da mídia no consumo alimentar infantil: uma revisão da literatura. Conselho Editorial, 140. 
Cruz, C. S. C., et al. (2015). OBESIDADE INFANTIL: ANÁLISE SOBRE A PRODUÇÃO CIENTÍFICA BRASILEIRA NO SCIELO. XVI ENANCIB. 3542.

Dias, P. C., et al. (2017). Obesidade e políticas públicas: concepções e estratégias adotadas pelo governo brasileiro. Cadernos de Saúde Pública, 33, e00006016,

Dietz WH. (2019). Childhood obesity. In: Shils ME, editor. Modern Nutrition in Health and Disease. Baltimore: Williams \& Williams, 1071-80.

Dipietro L. (2019). Physical activity in the prevention of obesity: current evidence and research issues. Med Sci Sports Exerc. 31(11 Suppl), S542-6.

Drummond, Porto Penna \& Juliana. (2012). Obesidade infantil: Abordagem na atenção primária. Universidade Federal de Minas Gerais. Curso deespecialização em atenção básica em saúde da família.

Figueiredo, Machado \& Abreu. (2012). A saúde na escola: um breve resgate histórico. Rev. Brasileira de Enfermagem.

Godinho, A. S. et al. (2019). Principais fatores relacionados ao sobrepeso e obesidade infantil. RENEF, 9(13), 27-39.

Golke, C. (2016). Obesidade infantil: Uma revisão de literatura. Universidade Federal de Santa Maria, Palmeiras das Missões.

Griggs S. \& Howarth D. (2011). Discourse and practice: using the power of well-being. Evid Policy, 7(2), 213-226.

Henriques, P., et al. (2014). A regulamentação da propaganda de alimentos no Brasil: convergências e conflitos de interesses. Cad Saude Publ, 30(6), 12191228.

Instituto Brasileiro De Geografia E Estatistica (IBGE). (2016). Pesquisa do estado nutricional de crianças, adolescentes e adultos no Brasil, 11.

Linhares, F. M. M., et al. Obesidade infantil: influência dos paissobre a alimentação e estilo de vida dos filhos. Temas em Saúde, 16(2), 460-48.

Lobstein, T., et al. (2015). Child and adolescent obesity: part of a bigger picture. Lancet, 385(9986), 2510- 2520.

Louzadam, M. L. C., et al. (2015). Consumption of ultra-processed foods and obesity in Brazilian adolescents and adults. Prev Med, 81, 9-15.

Martins, A. P. B., et al. (2013). Participação crescente de produtos ultraprocessados na dieta brasileira (1987- 2009). Rev Saude Publ, 47(4), 656-665.

Mattos, M. C. \& Cendón, B. V. (2014). Criação automática de uma base de citações para o SciELO a partir dos seus arquivos XML. Informação \& Tecnologia (ITEC), Marília/João Pessoa, 1(1), 42-67.

Miranda, J. M., et al. (2015). Prevalência de sobrepeso e obesidade infantil em instituições de ensino: públicas vs. privadas. Rev. Bras. Med. Esporte, 104-107.

Miziara \& Vectore. (2014). Excesso de peso em escolares: percepção e intercorrências nas escolas. Rev. Quadrimestral da Associação Brasileira de Psicologia Escolar e Educacional.

Nascimento, Brito \& Petriz. (2015). Promoção da Saúde como ferramenta de intervenção na obesidade infantil. Centro Universitário do Distrito Federal.

Nascimento, Schoeps, Souza \& Leone. (2011). Risco de sobrepeso e excesso de peso em crianças de préescolas privadas e filantrópicas. Rev. Associação médica brasileira.

Nunes, C. P., et al. (2019). Fator emocional na obesidade e transtornos de imagem. Revista de Medicina de Família e Saúde Mental, 1(2).

Oliveira, Braz, Nascimento \& Melo. (2016). Obesidade Infantil: Contribuição da enfermagem na prevenção. Rev. Humano Ser.

Porto, E.B.S, et al. (2015). School canteens in the Federal District, Brazil and the promotion of healthy eating. Rev Nutr, 28(1), 29-41.

Reis, Vasconcelos \& Barros. (2011). Políticas Públicas de nutrição para o controle da obesidade infantil. Rev. Paulista de Pediatria.

RNPI, Rede Nacional Primeira Infância. Plano Nacional da Primeira Infância. (2014). Obesidade na Primeira Infância. Ação Finalística Criança com Saúde. Fortaleza.

Rosaneli, Auler et al. (2012). Avaliação da prevalência e de determinantes nutricionais e sociais do excesso de peso em uma população de escolares: análise transversal em 5.037 alunos. Rev. Associação médica brasileira.

Santos, E. M. S., et al. (2020). Obesidade infantil: uma revisão bibliográfica sobre fatores que contribuem para a obesidade na infância. Revista Brasileira de Reabilitação e Atividade Física, Vitória, 9(1), 57-62.

Sahota P., Rudolf MCJ., Dixey R., Hill AJ., Barth JH. \& Cade J. (2017). Evaluation of implementation and effect of primary school based intervention to reduce risk factors for obesity. BMJ, 323, 1-4.

Silva, J. L. \& Bernardes, L. A. (2018). Relação entre consumo e obesidade infantil sob a ótica da análise do comportamento: revisão Narrativa. PretextosRevista da Graduação em Psicologia da PUC Minas, 3(6), 79-101.

Silva, L. R., et al. (2019). A influência dos pais no aumento do excesso de peso dos filhos: uma revisão de literatura/The influence of parents in increasing the excess of children's weight: a literature review. Brazilian Journal of Health Review, 2(6), 6062-6075. 
Research, Society and Development, v. 10, n. 6, e29610616443, 2021

(CC BY 4.0) | ISSN 2525-3409 | DOI: http://dx.doi.org/10.33448/rsd-v10i6.16443

Summerbell CD., Ashton V., Campbell KJ., Edmunds L., Kelly S. \& Waters E. (2018). Interventions for preventing obesity in children. Cochrane Database Syst Rev. Nutr, (3), CD001872.

Summerbell CD., Waters E., Edmunds L, OMeara S. \& Campbell K. (2017). Interventions for treating obesity in children [protocol]. In: The Cochrane Library, (1)

Swinburn, B., et al. (2015). Strengthening of accountability systems to create healthy food environments and reduce global obesity. Lancet, 385(9986), 25342545,13

Testa, W. L., Poeta, L. S. \& Da Silva Duarte, M. F. (2017). Exercício físico com atividades recreativas: uma alternativa para o tratamento da obesidade infantil. RBONE-Revista Brasileira de Obesidade, Nutrição e Emagrecimento, 11(62), 49-55.

Wright CM., Parker L., Lamont D. \& Craft AW. (2018). Implications of childhood obesity for adult health: findings from thousand families cohort study. BMJ, 323(7324), 1280-4.

Wilkin TJ., Metcalf BS., Murphy MJ., Kirkby J., Jeffery NA. \& Voss LD. (2016). The relative contributions of birth weight, weight change, and current weight to insulin resistance in contemporary 5-yearolds: The EarlyBird Study. Diabetes. 51(12), 3468-72. 